\title{
BMJ Open Effect of reactive balance training on physical fitness poststroke: study protocol for a randomised non- inferiority trial
}

\author{
Azadeh Barzideh, ${ }^{1,2}$ Susan Marzolini, ${ }^{2}$ Cynthia Danells, ${ }^{2,3}$ David Jagroop, ${ }^{2}$ \\ Andrew H Huntley, ${ }^{2}$ Elizabeth L Inness, ${ }^{2,3}$ Sunita Mathur, ${ }^{2,3}$ George Mochizuki, ${ }^{4}$ \\ Paul Oh, ${ }^{2}$ Avril Mansfield (i) ${ }^{2,3,5}$
}

To cite: Barzideh A, Marzolini S, Danells $\mathrm{C}$, et al. Effect of reactive balance training on physical fitness poststroke: study protocol for a randomised non-inferiority trial. BMJ Open 2020;10:e035740. doi:10.1136/ bmjopen-2019-035740

- Prepublication history and additional material for this paper are available online. To view these files, please visit the journal online (http://dx.doi org/10.1136/bmjopen-2019035740).

Received 13 November 2019 Revised 12 May 2020 Accepted 18 May 2020

Check for updates

(C) Author(s) (or their employer(s)) 2020. Re-use permitted under CC BY-NC. No commercial re-use. See rights and permissions. Published by BMJ.

${ }^{1}$ Rehabilitation Sciences Institute, University of Toronto, Toronto, Ontario, Canada

${ }^{2}$ Toronto Rehabilitation Institute, University Health Network, Toronto, Ontario, Canada ${ }^{3}$ Physical Therapy, University of Toronto, Toronto, Ontario, Canada

${ }^{4}$ Kinesiology, York University, Toronto, Ontario, Canada

${ }^{5}$ Evaulative Clinical Sciences, Hurvitz Brain Sciences Program, Sunnybrook Research Institute, Toronto, Ontario, Canada

Correspondence to

Dr Avril Mansfield;

avril.mansfield@uhn.ca

\section{ABSTRACT}

Introduction Regular exercise is essential in the chronic phase of stroke recovery for improving or maintaining function, and reducing the risk of a second stroke. To achieve these goals, multiple components of fitness should be targeted with poststroke exercise, including aerobic capacity, strength and balance. However, following the recommended frequency and duration of each component separately can take a long time and lead to fatigue in people with stroke. Therefore, finding types of exercise that target multiple components of fitness all together is valuable.

Reactive balance training (RBT) is a novel type of exercise where individuals repeatedly lose their balance in order to practise balance reactions. When people do RBT, they increase their heart rate and exert forces with their leg muscles which could improve aerobic fitness and muscle strength, respectively. This means that RBT could have the potential to improve multiple components of fitness, simultaneously.

Methods and analysis This is a randomised controlled non-inferiority trial with internal pilot study. Participants with chronic stroke will be randomly assigned to one of two groups: (1) RBT or (2) aerobic and strength training (AST). Participants in both groups will complete 1 hour of exercise, three times/week for 12 weeks. The primary objective is to determine the effect of RBT on aerobic capacity and knee muscles' strength. The secondary objective is to determine the effects of RBT and AST on balance control and balance confidence. We expect to find that RBT is superior to AST in terms of improving balance control and balance confidence, yet not inferior to AST in terms of its effects on aerobic capacity and strength. Ethics and dissemination Research ethics approval has been received. Results will be disseminated directly to study participants at the end of the trial, and to other stakeholders via publication in a peer-reviewed journal. Trial registration number NCT04042961.

\section{INTRODUCTION}

\section{Background and rationale}

Regular exercise is essential in the chronic phase of stroke recovery for promoting ongoing recovery, improving or maintaining
Strengths and limitations of this study

- The proposed amount of training is similar to that of strength and cardiorespiratory training poststroke and exceeds that of previous studies of perturbation training poststroke.

- The internal pilot component of this study gives us the opportunity to evaluate whether progressing with the study helps us to address the primary and secondary objectives.

- This trial is assessor blinded; participants and the therapist delivering the intervention cannot be blinded to intervention allocation, which potentially introduces a source of bias.

physical function and reducing the risk of having another stroke. ${ }^{12}$ There are multiple components of fitness that should be targeted with poststroke exercise, including cardiorespiratory fitness, muscle strength and neuromotor control (eg, balance control). ${ }^{23}$

People with stroke have difficulty meeting the recommended exercise guidelines. ${ }^{2}$ If each component of fitness is targeted separately (eg, brisk walking for cardiorespiratory fitness, resistance training for strength and tai chi for balance), then following the recommended frequency and duration of each type of exercise could require several hours of exercise per week. ${ }^{2}$ Lack of time and fatigue are frequently reported as barriers to exercise poststroke. ${ }^{4-10}$ Therefore, it is important to identify types of exercise that target multiple components of fitness concurrently in order to improve efficiency of poststroke exercise programmes.

Reactive balance training (RBT) is a novel type of exercise where individuals repeatedly experience postural perturbations in order to practise balance reactions. ${ }^{11}$ RBT improves aspects of neuromotor control 
(balance); RBT is the only type of exercise that has shown the ability to improve control of reactions that are essential to prevent falls following a loss of balance (ie, 'reactive balance control'), ${ }^{12-17}$ and can also improve balance control during voluntary movement (ie, 'anticipatory balance control') in people with stroke. ${ }^{17}$ RBT involves executing rapid whole-body movements in quick succession and exerting large and rapid eccentric forces to halt the falling centre of mass. The whole-body movements are similar to the repetitive, dynamic movements that form part of aerobic exercise training (eg, brisk walking or cycling). This dynamic and whole-bodied nature of RBT may have the potential to improve strength and cardiorespiratory fitness, in addition to the benefit to balance control.

While previous studies have found that RBT can improve balance control, ${ }^{17}{ }^{18}$ determining whether it can also improve muscle strength and cardiorespiratory fitness could be advantageous in the clinical setting. If RBT is beneficial across several domains of fitness, clinicians could use it as an alternative training modality for targeting muscle strength and aerobic capacity in addition to its positive effects on balance control. Additionally, patients who had a stroke who commonly face barriers to exercise such as fatigue and lack of time can use their time more efficiently by performing RBT as an alternative to aerobic and strength exercises to benefit different components of fitness.

\section{Objectives}

The primary purpose of this study is to determine the effect of RBT on two major components of fitness among individuals with chronic stroke: cardiorespiratory fitness and isokinetic muscle strength. Our primary hypothesis is that improvements in cardiorespiratory fitness (peak oxygen consumption) and lower extremity muscle strength (peak force production during knee extension and flexion) following RBT will be not inferior to the improvements following aerobic and strength training (AST) programme. The secondary purpose of this study is to determine the effect of RBT and AST on balance control and balance confidence. We hypothesise that, compared with AST, RBT will lead to greater increases in: 1. Anticipatory balance control (Berg Balance Scale, BBS).

2. Reactive balance control ('reactive control' subscale of the mini-Balance Evaluation Systems Test (miniBEST)).

3. Balance confidence (Activity-specific Balance Confidence (ABC) questionnaire).

\section{Trial design}

This is an assessor-blinded randomised non-inferiority trial with an internal pilot study. Research activities will take place at the Toronto Rehabilitation Institute and the University of Toronto. For the internal pilot study, we will initially recruit 20 participants with chronic stroke and randomly assign them to one of two groups: (1) RBT or (2)
AST. Cardiorespiratory fitness, lower extremity strength, balance control and balance confidence will be measured preintervention and postintervention (figure 1). We will calculate the final target sample size using the variability observed in this internal pilot.

\section{Patient and public involvement}

This study was designed without patient involvement. Patients were not invited to comment on the study design and were not consulted to develop patient-relevant outcomes. Some trial design elements were informed by participant feedback from our previous RBT study. ${ }^{17}$ Patients were not invited to contribute to the writing or editing of this document for readability or accuracy.

\section{METHODS: PARTICIPANTS, INTERVENTIONS AND OUTCOMES Eligibility criteria}

Community-dwelling adults with chronic stroke $(>6$ months poststroke) will be recruited. All participants must be able to stand independently without upper limb support for $>30 \mathrm{~s}$ and tolerate at least 10 postural perturbations while wearing a safety harness.

The following exclusion criteria will be used:

- A height $>2.1 \mathrm{~m}$ tall and/or weighing $>150 \mathrm{~kg}$ (limits of the safety harness system).

- Other neurological condition(s) that could affect balance control (eg, Parkinson's disease).

- Lower extremity amputation.

- Cognitive, language and/or communication impairments affecting understanding instructions.

- Recent ( $\leq 6$ months) significant illness, injury or surgery.

- Severe osteoporosis (defined by diagnosis of osteoporosis with fracture).

- Severe uncontrolled hypertension and/or uncontrolled/insulin-dependent diabetes.

- Active diabetic retinopathy or moderate or worse nonproliferative retinopathy.

- Symptomatic hernia.

- Contraindications to exercise testing, including symptomatic aortic stenosis, complex life-threatening arrhythmias, unstable angina or orthostatic blood pressure decrease of $>20 \mathrm{~mm} \mathrm{Hg}$ with symptoms. ${ }^{19}$

- Acute or chronic illness or injury likely to be exacerbated by exercise (eg, recent lower extremity fracture).

- Currently attending inpatient or outpatient physiotherapy, in which they receive aerobic exercise, balance training and/or lower limb strength training.

- Significant exercise participation: current physical activity levels that meet the recommended guidelines (at least 150 min of moderate to vigorous or at least 75 min of vigorous physical activity/week) as calculated using the moderate and vigorous components of the Leisure Time Exercise Questionnaire ${ }^{20}$ in the month prior to starting the study.

- Received RBT $<1$ year previously. 


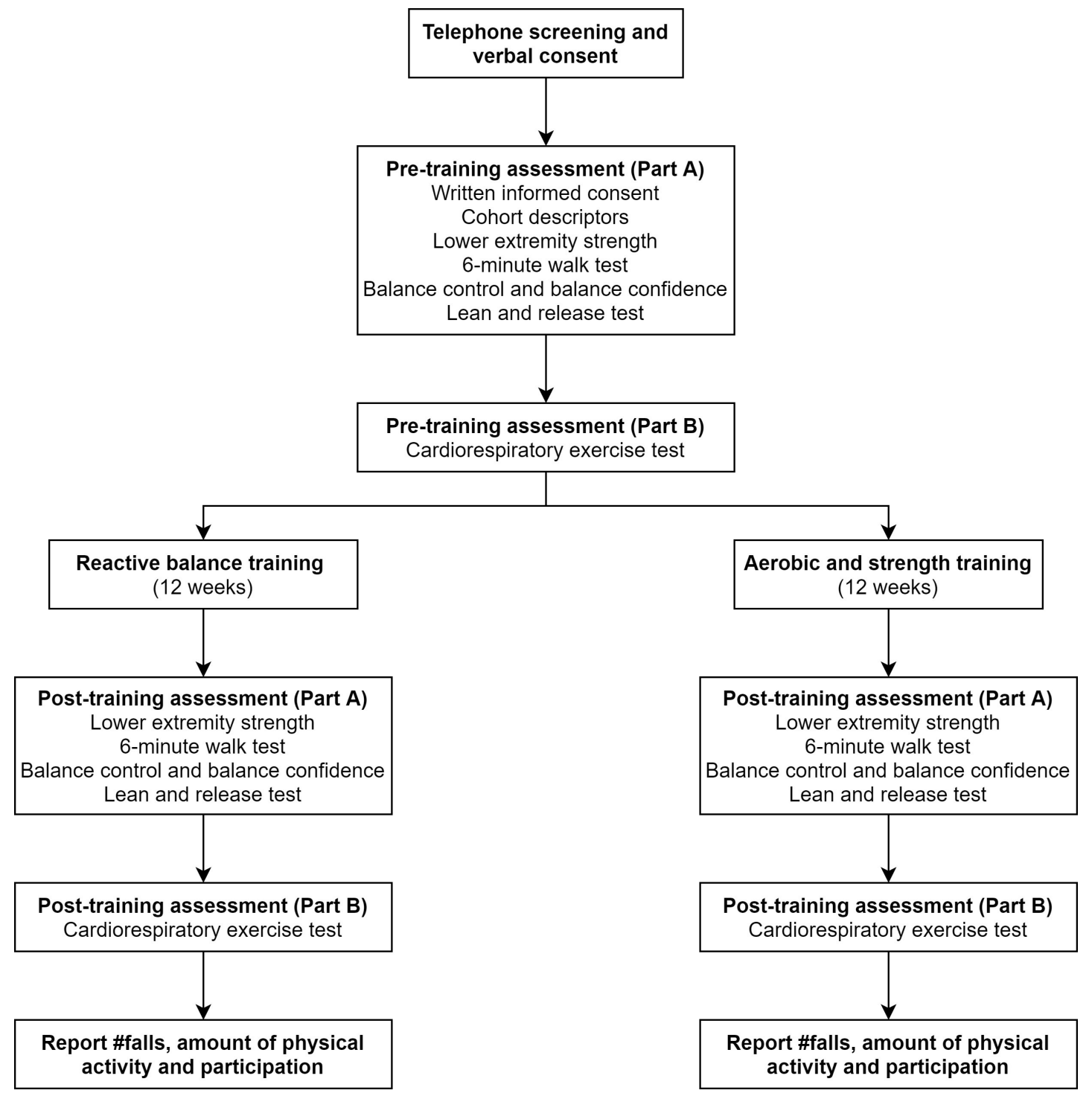

Figure 1 Participant flow through the study.

\section{Interventions}

Participants will complete three 60 min exercise sessions per week for 12 weeks. The proposed amount of training is similar to that of strength and cardiorespiratory training poststroke in terms of frequency, duration and intensity ${ }^{19}$ and exceeds that of previous studies of RBT poststroke. ${ }^{1121} 22$ Interventions will be completed in a research laboratory and gym within a rehabilitation hospital on a 1:1 basis (ie, one physiotherapist per participant). The same physiotherapist will administer both interventions. Interventions will follow a general guide but will be tailored to the individual participants' ability. For example, in the RBT programme, if 'inability to step with the affected leg' is identified as an impairment, voluntary tasks may be altered to encourage more opportunities to react with the affected leg.

Participants will wear a data-logging heart rate monitor (Suunto Smart Sensor, Vantaa, Finland) during exercise to measure the acute cardiorespiratory response. Blood glucose $(\mathrm{mmol} / \mathrm{L})$ and/or blood pressure $(\mathrm{mm} \mathrm{Hg})$ will be measured pretraining and post-training for those at risk for hypoglycaemia (individuals with insulin-independent and controlled diabetes) and/or with elevated resting blood pressure, respectively. To help alleviate barriers to attending the programme we will reimburse participants for their travel expenses.

\section{Group 1: RBT}

In the development of our RBT programme, all the principles of motor learning (ie, practice variability, practice order, feedback, guidance, instruction and focus of attention) and exercise physiology (ie, overload, adaptation, progression, individualisation and specificity) are taken into account. ${ }^{2123}$ In order to individualise the RBT programme to each participant, the physiotherapist will identify participant-specific impairments in the control of reactive stepping using the lean-and-release assessment (performed in the baseline assessment session) ${ }^{18}$ and through further assessment during the first training session. The physiotherapist will 
then design the programme in a way that targets participants' specific impairments.

The RBT programme will include external and internal postural perturbations. External perturbations are caused by an external force, such as a push or pull from the physiotherapist. Internal perturbation occurs when the participant performs a challenging task, and is unable to control the centre of mass base of support relationship. This can happen when participants are performing voluntary motor tasks that are more challenging than their current anticipatory balance control ability; for example, tandem walking. In general, for all the participants, the difficulty of the tasks will be set such that participants will 'fail' to recover balance $\sim 50 \%$ of the time; 'failure' is defined as the need for external assistance either from the physiotherapist or the overhead harness, or taking more than two steps to regain stability. Participants will wear a safety harness during all RBT training sessions. In our previous randomised controlled trial in individuals with chronic stroke, participants experienced response failures, on average, $57 \%$ of the time. ${ }^{17}$ The physiotherapist can increase the task difficulty by increasing the magnitude or changing the direction or timing of the postural perturbation, by including additional cognitive or movement tasks ${ }^{23}$ or by adding sensory or environmental challenges (eg, eyes closed, use of obstacles). Each session in the RBT programme will include a 5 min warm-up, at least 60 perturbations and a $5 \mathrm{~min}$ cool-down. In order to induce these 60 internal or external perturbations, each session will include the performance of 10 different voluntary tasks similar to those described in Mansfield et al (eg, standing and weight shifting, or stepping) ${ }^{17}$ Regardless of voluntary task, the magnitude, direction and timing of the external perturbation, delivered during performance of the voluntary task, will be chosen to address each participant's balance-specific impairment (eg, use of non-preferred limb, step clearance, and so on). There will be six induced perturbations per task. The physiotherapist will record the number of perturbations that a participant experiences during the session. Our previous study in individuals with chronic stroke showed that the participants were able to complete, on average, 55 perturbations in 1 hour sessions. ${ }^{17}$

\section{Group 2: AST}

Each AST session will consist of up to $30 \mathrm{~min}$ of aerobic training and 30 min of strength training. Each session will start with a 5 min warm-up on the aerobic training modality at a low to moderate intensity $(30 \%-60 \%$ heart rate reserve (HRR)). Participants will then complete the AST, as described below, and will finish the training session with 5 min cool-down which includes static stretching, range of motion and light-intensity aerobic activities, for example, walking at a comfortable pace. ${ }^{19}$

\section{Aerobic training}

Continuous treadmill walking will be the primary mode of aerobic training. For those unable to maintain their target training heart rate for $\geq 5-10$ min during walking, a combination of modalities will be used (eg, treadmill walking plus, recumbent/upright cycling or recumbent stepping). ${ }^{24}$ However, the ultimate goal is to progress patients to a faster walking pace and longer duration of walking on treadmill rather than cycling. From the baseline cardiopulmonary exercise test (CPET) results, the heart rate at the ventilatory threshold $\left(\mathrm{VO}_{2 \mathrm{VT}}\right)$ will be used to prescribe aerobic exercise intensity. ${ }^{25}$ In the absence of a discernible $\mathrm{VO}_{2 \mathrm{VT}}$, a combination of the following methods will be used: $60 \%-80 \%$ of HRR or peak oxygen uptake $\left(\mathrm{VO}_{\text {2peak }}\right)$, and/or rating of perceived exertion (RPE) of 11-16 on the Borg $^{26} 6-20$ scale. ${ }^{19}$ The goal will be to progress participants to 20-30 min of exercise, three times per week. Prescriptions will be initially progressed by increasing exercise duration to $\geq 20$ min to a maximum of $30 \mathrm{~min}$ and then increasing intensity to target heart rate (maximal duration $30 \mathrm{~min}$ ). Heart rates measured during exercise and communication with the physiotherapist will aid in deciding when to increase the prescription.

\section{Strength training}

Participants will be prescribed two sets of eight exercises per session, including lunge, heel raise, ankle dorsiflexion, knee extension and flexion, abdominal curl-up, wall push-up and bicep curl. If participants are unable to do a leg curl in standing, they will be asked to sit and perform the exercise. Each set consists of $8-10$ repetitions. The one-repetition maximum (1RM) test will be performed on affected and non-affected sides separately, which is the greatest amount of weight lifted for a single repetition. ${ }^{19}$ For this purpose different types of weights will be used: dumbbells, stacked weights, resistance bands and a weighted vest. The type of weight used will be determined by the participant's limb control and functional ability. After three repetitions of a warm-up weight, a slightly heavier weight will be attempted one time following a 2 min recovery period. One repetition at a heavier weight will be attempted every 2 min until the participant is unable to lift the weight with proper technique. 1RM testing will occur at baseline, and repeated at weeks 3, 6 and 10 and the weight load will be adjusted to $70 \%$ of 1RM starting at week 3. Between 1RM testing, progressions during the training sessions will be made in the following steps: (1) gradually progressing from 8 to 10 repetitions; (2) increasing resistance by $1.6-5 \mathrm{~kg}$ or one exercise band level while reducing repetitions to 8; (3) repeating this process (ie, increasing from 8 to 10 repetitions). Progression of movement velocity to optimise dynamic muscle strength will be introduced after $\geq 4$ weeks of training. Participants will initially be prescribed a weight load equivalent to $50 \%-60 \%$ of $1 \mathrm{RM}$ assessed at baseline and/or a resistance rated as 13-16 on the RPE scale ${ }^{26}$ on the last repetition of the set. ${ }^{24}$

\section{Outcomes}

Outcome measures and assessment time points are summarised in table 1 and figure 1.

Primary outcome: cardiorespiratory fitness

A symptom-limited CPET will be performed on a semirecumbent cycle ergometer with specialised pedals to 
Table 1 Summary of outcome measures and assessment time points

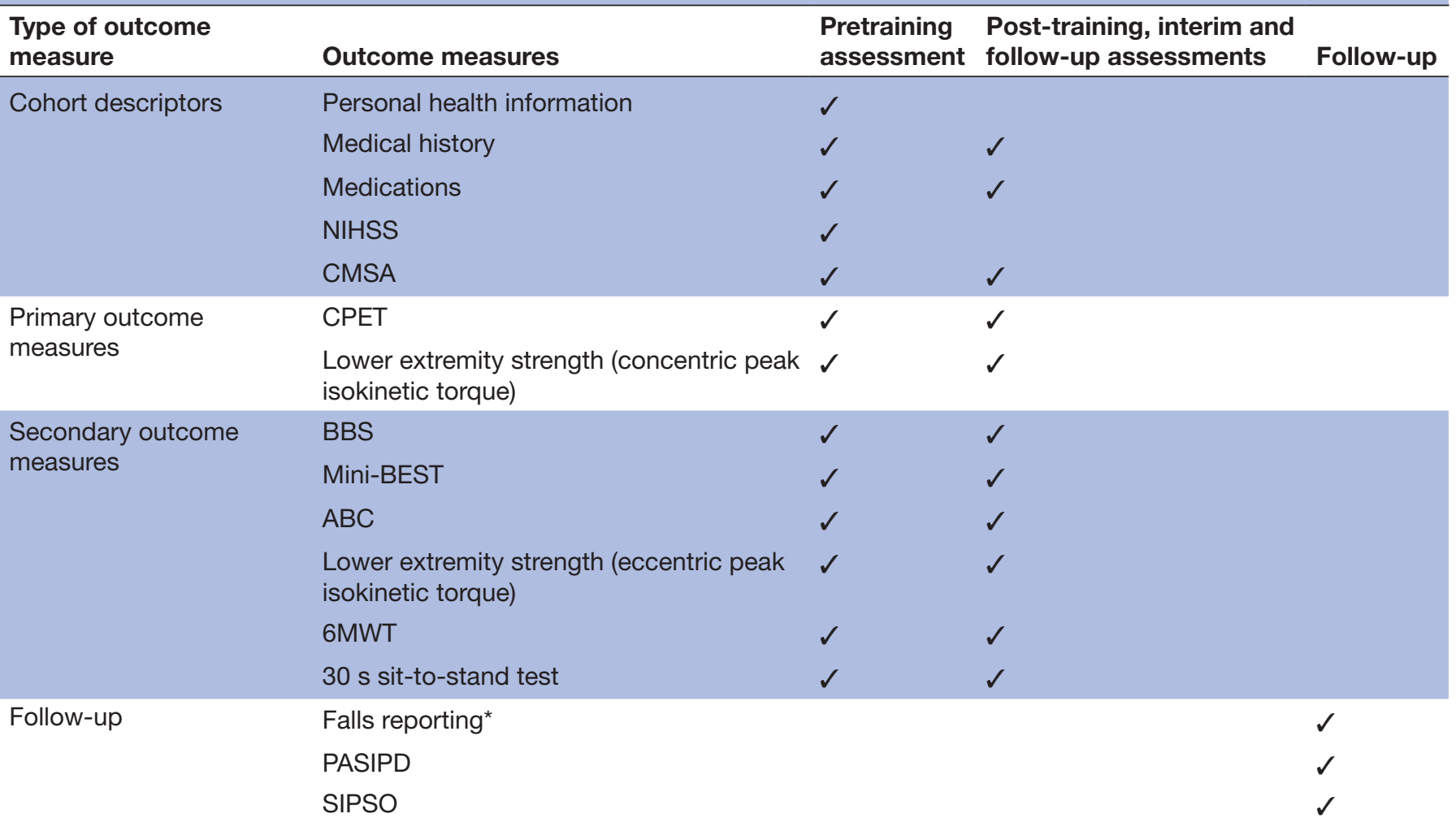

${ }^{*}$ Reported continuously throughout the 12-month follow-up period.

ABC, Activity-specific Balance Confidence questionnaire; BBS, Berg Balance Scale; CMSA, Chedoke-McMaster Stroke Assessment; CPET, cardiopulmonary exercise test; Mini-BEST, mini-Balance Evaluation Systems Test; 6MWT, 6 min walk test; NIHSS, National Institutes of Health Stroke Scale; PASIPD, Physical Activity Scale for Individuals with Physical Disabilities ; SIPSO, Subjective Index of Physical and Social Outcome.

secure feet (Ergoline, Ergoselect 1000, Blitz, Germany), upright cycle (Ergoselect 200P, Germany) or a treadmill depending on balance and ability to control the leg/foot position in pedals. ${ }^{27} \mathrm{~A}$ treadmill will be used for the CPET if participants meet the following criteria in the first assessment session: (1) Chedoke-McMaster Stroke Assessment (CMSA) leg and foot scores $\geq 4$; (2) 6 min walk test $(6 \mathrm{MWT}) \geq 144 \mathrm{~m}$; and (3) able to walk on the treadmill at least $3 \mathrm{~min}$ at the speed of $1.7 \mathrm{mph}$. If participants do not meet these criteria, they will be assigned to semirecumbent/upright cycle. Participants' preference for using a treadmill or cycle will also be taken into account.

The CPET will be medically supervised. It will be conducted on the same modality on subsequent assessments and at the same time of day as the training sessions to minimise the effects of heart rate altering medication on exercise prescription. If treadmill is used as the modality for CPET, a modified Bruce protocol will be employed. ${ }^{28}$ This protocol is usually used for patients who are predicted to have poor exercise capacity. The difference between the Bruce and the modified Bruce protocol is in two warm-up stages which are added before the first stage of Bruce protocol; that is, the third stage of modified Bruce protocol corresponds to the first stage of Bruce protocol. Therefore, the first three stages of modified
Bruce protocol have a constant speed of $1.7 \mathrm{mph}$ and the inclination increases by $5^{\circ}$ every $3 \mathrm{~min} .{ }^{29}$ If an upright cycle or recumbent cycle are the chosen modalities for CPET, workload will be increased by either 8.3 or 16.7 (W) every minute at a pedalling rate of $60 \mathrm{rpm}$ (revolutions per minute), the aim being to achieve test durations of between 8 and 12 min. ${ }^{27}$ Breath-by-breath gas samples will be collected via calibrated metabolic cart (SensorMedics Vmax Encore, San Diego, California, USA) to determine $\mathrm{VO}_{2 \text { peak }}$. Twelve-lead ECG, RPE and blood pressure will be monitored throughout. The CPET has excellent testretest reliability in people with chronic stroke (intraclass correlation coefficient $($ ICC $)=0.93) .^{30}$

Oxygen uptake at the ventilatory threshold $\left(\mathrm{VO}_{2 \mathrm{VT}}\right)$ will be determined by a combination of the V-slope method and the ventilatory equivalent method ${ }^{31}$ as previously described. ${ }^{27} 32$ The V-slope method is defined as the point of departure from linearity of carbon dioxide output plotted against oxygen uptake. The ventilatory equivalent method is defined as the level corresponding to the rise in ventilatory equivalent of oxygen that occurs when the ventilatory equivalent of carbon dioxide remains relatively constant. The $\mathrm{VO}_{2 \mathrm{VT}}$ is considered a valid measure of cardiorespiratory fitness, demonstrating high interrater reliability in the stroke population $(\mathrm{ICC}=0.93) .{ }^{33}$ 


\section{Primary outcome: lower extremity strength}

Peak isokinetic torque will be measured using a Biodex System 4 Pro dynamometer (Biodex Medical Systems, New York, USA). Participants will be seated in the chair (hips at approximately $90^{\circ}$ ) with the axis of rotation of the dynamometer aligned to the lateral femoral condyle. Shoulder straps will secure the torso and a thigh strap over the active leg will minimise compensatory movements during testing. The inactive leg will be positioned at $90^{\circ}$ knee flexion and put on a footrest below the seat. Peak isokinetic muscle torque at an angular velocity of $60^{\circ} / \mathrm{s}^{34-36}$ will be assessed. While Rabelo and colleagues reported high reliability in muscle strength testing for different angular velocities and types of contraction (from 30 to $240^{\circ} / \mathrm{s}$ for concentric and from 30 to $180^{\circ} / \mathrm{s}$ for eccentric contraction), ${ }^{37}$ they did not address any potential injury at higher speeds. In this study, $60^{\circ} / \mathrm{s}$ was chosen as many studies ${ }^{38-42}$ have used this velocity in patients who had a stroke and have reported high to very high reliability in both knee extension and flexion. Additionally, considering the high impact of eccentric contractions on muscles and its risks, ${ }^{43}$ choosing a lower angular velocity can minimise the risk of muscle injury. Three warm-up contractions will be performed at $\sim 50 \%$ of perceived maximum effort for knee extension and flexion before starting the five maximal trials for knee extension and flexion in each concentric contraction and eccentric contraction. All participants will start the muscle strength testing with the concentric contractions of knee flexors and extensors on the less affected leg. They will then complete eccentric contractions of knee flexors and extensors on the same side. The same process will be repeated on the more affected leg after a $5 \mathrm{~min}$ rest in which the research assistant will set up the device for the other side. Eccentric contractions of knee flexors and extensors will be performed between $20^{\circ}$ and $90^{\circ}$ knee flexion to protect the knee from injuries to prevent potential injuries related to the knee hyperextension. ${ }^{41}$ The torque limit for the eccentric contractions will be chosen from three different values of 100,135 or 200 $\mathrm{Nm}$ based on each individual's relative muscle strength assessed with $30 \mathrm{~s}$ sit-to-stand test which is a functional measure of strength, CMSA score which is indicative of their stage of motor recovery and, finally, their walking disability (if any). A $1 \mathrm{~min}$ rest will be given between trials to minimise the effect of fatigue. The average of three maximal torques among the five trials of concentric contractions for each hamstring and quadriceps will be considered as their concentric peak isokinetic torque. The same method will be used to calculate the eccentric peak isokinetic torque for each hamstring and quadriceps. The task will be performed for both legs. A single value will be obtained for knee flexion and knee extension of the paretic and non-paretic legs.

\section{Secondary outcomes}

Balance will be assessed using the $\mathrm{BBS}^{44}$ and the miniBEST. $^{45}$ The BBS is a functional measure of balance with 14-item observational rating scale. Participants will be asked to perform each of the 14 tasks. Their ability to perform the task will be rated on a scale from 0 to 4 . The BBS shows good internal consistency (Cronbach's $\alpha=0.92-0.98)$ and good inter-rater (ICCs=0.95-0.98), intrarater $(\mathrm{ICCs}=0.97)$ and test-retest reliability $(\mathrm{ICC}=0.98)$ in the stroke population. ${ }^{46}$ The mini-BEST is a 14 -item observational rating scale which assesses systems underlying balance control, including reactive balance control and dynamic stability during walking. ${ }^{45}$ The mini-BEST has good inter-rater $(\mathrm{ICC}=0.96)$ and intrarater reliability $(\mathrm{ICC}=0.97)$ among individuals with chronic stroke. ${ }^{45} 47$

The ABC scale ${ }^{48}$ is a 16 -item questionnaire used to assess balance confidence during activities of daily living before and after training. This questionnaire asks participants to rate how confident they are while performing 16 everyday tasks using a scale from $0 \%$ to $100 \%$. The ABC shows good internal consistency (Cronbach's $\alpha=0.94$ ) and test-retest reliability $(\mathrm{ICC}=0.85)$ in individuals with stroke. $^{49}$

The $6 \mathrm{MWT}$ will be conducted as a measure of functional capacity and endurance. ${ }^{50}$ This test asks participants to walk as far as possible for 6 min on a $30 \mathrm{~m}$ path. ${ }^{51} 6 \mathrm{MWT}$ has excellent test-retest reliability $(\mathrm{ICC}=0.99)$ in chronic stroke. ${ }^{3052}$ Functional strength will be assessed using the $30 \mathrm{~s}$ sit-to-stand test. ${ }^{53}$ For this test, the participant will be asked to sit on a $43 \mathrm{~cm}$ high chair ${ }^{54}$ which will be placed against a wall for stability. They will be asked to rise to a full stand, and then return to a fully seated position, as many times as possible in $30 \mathrm{~s}$ without the use of upper limbs. Previous studies have shown an excellent interrater $\left(\mathrm{ICC}_{1,2}=0.88\right.$ and 0.94$)$ and intrarater $\left(\mathrm{ICC}_{1,2}=0.87\right.$ and 0.91$)$ reliability for $30 \mathrm{~s}$ sit-to-stand test in the stroke population. ${ }^{55}$ These two functional tests will help us investigate whether the effects of the exercise programmes on endurance and muscle strength will also translate into functional activities.

Participants will complete a 12-month falls monitoring period after completing the initial training period. For this purpose, they will be provided stamped, addressed postcards containing a calendar to record falls, which they will be asked to complete daily. A fall is defined as 'an event that results in a person coming to rest unintentionally on the ground or other lower level'. ${ }^{56}$ Participants will return each postcard to the research team fortnightly. Monthly study newsletters which contain health-related articles of interest, as well as a reminder to complete the event tracking, will be mailed to our participants. The research assistant will contact the participants via telephone, if they have not completed this event tracking within 2 weeks. In this telephone call, the research assistant will try to ascertain if the participant has experienced a fall in the previous 2 weeks. This method is considered the 'gold standard' for falls reporting. ${ }^{57}$ Participants who report a fall on the calendar will be contacted by the research assistant to complete a short questionnaire in order to determine the cause and consequences of the fall. The number of falls together with the falls and the 
$\mathrm{ABC}$ questionnaires will give us a better understanding of the participants' fear of falling and how the falls usually happen in stroke population and whether they change with exercise interventions.

Physical activity and participation will be evaluated with the Physical Activity Scale for Individuals with Physical Disabilities (PASIPD) ${ }^{58}$ and the Subjective Index of Physical and Social Outcome (SIPSO), ${ }^{59}$ at three time points during the 12-month follow-up. Regular administration of the questionnaires will provide an estimate of physical activity and participation over the duration of the follow-up period. The PASIPD is a 13-item questionnaire in which participants are asked to indicate the frequency and duration of recreational, household and occupational physical activities completed in the previous 7 days. The PASIPD has been validated within a group of individuals with various physical disabilities, including those with stroke, showing good test-retest reliability $(\mathrm{r}=0.77)$ and criterion validity when compared with accelerometer-based activity monitoring $(\mathrm{r}=0.30) .{ }^{60}$ The SIPSO is a 10 -item questionnaire that evaluates the physical and social integration and participation in 'normal' daily life. ${ }^{61}$ The SIPSO has good internal consistency (Cronbach's $\alpha=0.92$ ) and test-retest reliability (ICC $=0.91$ ) among those with stroke. ${ }^{59}$ Obtaining information about the amount of physical activity and social participation of our participants can help us have a better picture of the long-term effect of our interventions as a successful rehabilitation should ultimately result in quality of life and amount of participation in the community that is close to that before the stroke. ${ }^{62}$

\section{Cohort descriptors}

The following demographic and medical information will be recorded at the time of study enrolment in order to characterise the study cohort: age, sex, height, weight, waist circumference, time since stroke, lesion location medical history, prescription medications, the National Institutes of Health Stroke Scale (NIHSS) ${ }^{63}$ and CMSA ${ }^{64}$ foot and leg scores. The NIHSS is an 11-item scale that provides a gross measure of the effects and severity of stroke. ${ }^{64}$ The NIHSS has good intrarater $($ ICCs $=0.93)$ and inter-rater $($ ICCs $=0.95)$ reliability ${ }^{65}$ The CMSA assigns a score according to the level of motor recovery (ie, there are seven levels in total) in the foot and leg and is frequently used to evaluate motor impairment poststroke in clinical settings. The CMSA foot and leg scores have good intrarater $(\mathrm{ICCs}=0.94-0.98)$ and inter-rater $(\mathrm{ICCs}=0.85-0.96)$ reliability. ${ }^{64}$ If participants were previously patients at the Toronto Rehabilitation Institute, their medical history (ie, premorbid medical history, date of stroke and lesion location) will be confirmed with chart review.

\section{Sample size}

Following completion of the internal pilot study (20 participants), sample size for a non-inferiority trial with continuous outcome will be calculated using the formula below and based on our primary outcome measures ${ }^{6667}$ :
Table 2 Sample size estimation

\begin{tabular}{|c|c|c|c|}
\hline Outcome measure & $\begin{array}{l}\text { Variance } \\
\text { estimate }\end{array}$ & SEM & $\begin{array}{l}\text { Initial } \\
\text { sample size } \\
\text { estimate }\end{array}$ \\
\hline $\mathrm{VO}_{2 \text { peak }}(\mathrm{mL} / \mathrm{kg} / \mathrm{min})$ & 1.69 & 1.0 & 29 \\
\hline $\begin{array}{l}\text { Non-paretic limb knee } \\
\text { extension strength (Nm) }\end{array}$ & 72.3 & 10.6 & 11 \\
\hline $\begin{array}{l}\text { Paretic limb knee extension } \\
\text { strength }(\mathrm{Nm})\end{array}$ & 43.6 & 12.4 & 5 \\
\hline $\begin{array}{l}\text { Non-paretic limb knee } \\
\text { flexion strength }(\mathrm{Nm})\end{array}$ & 17.6 & 7.4 & 5 \\
\hline $\begin{array}{l}\text { Paretic limb knee flexion } \\
\text { strength }(\mathrm{Nm})\end{array}$ & 16.8 & 10.1 & 3 \\
\hline
\end{tabular}

The non-inferiority limit is the SEM. The sample size is the number of participants required per group.

SEM, SE of measurement.

$$
\mathrm{n}=\frac{2\left(\mathrm{Z}_{1-\beta}+\mathrm{Z}_{1-\alpha}\right)^{2} \sigma^{2}}{\left(\mathrm{~d}_{\mathrm{NI}}\right)^{2}}
$$

where $n$ is the number of participants per group, $Z_{1-\beta}$ is the $\mathrm{Z}$ statistic corresponding to probability of a type II error ( $\beta=0.1), Z_{1-\alpha}$ is the $Z$ statistic corresponding to the probability of a type I error $(\alpha=0.05), \sigma^{2}$ is the estimated population variance for the primary outcome $(\mathrm{s})$ and $\mathrm{d}_{\mathrm{NI}}$ is the non-inferiority limit. The non-inferiority limit is the maximum acceptable difference between the experimental (RBT) and comparison (AST) interventions. It is recommended to set the non-inferiority limit below the minimal clinically important difference ${ }^{68}$ such that, after intervention, the two groups are expected to differ by less than the clinically important difference. We set the non-inferiority limit at $100 \%$ of the SE of measurement (SEM) from previous studies of test-retest reliability of cardiorespiratory fitness $^{30}$ and lower extremity strength ${ }^{36}$ poststroke. The variance has initially been estimated from previous studies of interventions to improve aerobic capacity $^{69}$ and lower extremity strength ${ }^{35}$ in chronic stroke. Variances (from previous research), SEMs, noninferiority limits and associated sample sizes for each of the primary outcomes are outlined in table 2 . Therefore, our current plan is to recruit 35 participants ${ }^{30} 353669$ per group to account for a $20 \%$ rate of withdrawal. However, the variance from the internal pilot study will be used to re-estimate the sample size.

\section{Recruitment}

Several strategies will be used to identify participants, including: (1) inviting participants of previous studies conducted by the investigators; (2) requesting referrals from stroke rehabilitation programmes at the Toronto Rehabilitation Institute; (3) research participant databases at the Toronto Rehabilitation Institute; and (4) placing advertisements in the community (eg, in health centres or local newspapers). 


\section{METHODS: ASSIGNMENT OF INTERVENTIONS Intervention allocation}

Participants will be assigned using blocked stratified randomisation with allocation concealment to one of two training groups: (1) RBT or (2) AST. The block size will be 4,6 or 8 . There will be four strata based on two stratification factors: BBS score (two levels: $\leq 46$ vs $>46$ ) and baseline $\mathrm{VO}_{2 \text { peak }}$ (two levels: $<15 \mathrm{~mL} / \mathrm{kg} / \mathrm{min}$ vs $\geq 15 \mathrm{~mL} / \mathrm{kg}$ / $\min )$. A previous study found that BBS scores and baseline $\mathrm{VO}_{\text {2peak }}$ predicted response to aerobic exercise among individuals with chronic stroke. ${ }^{70}$ Therefore, stratification on these factors will ensure that the two groups are approximately equivalent on these important prognostic variables. Group allocation will be performed centrally by the principal investigator (PI) who will not be involved in recruiting, assessments or administering the interventions. The random allocation sequence will be computer generated. On completion of the baseline assessment, information required for randomisation (BBS score and $\mathrm{VO}_{2 \text { peak }}$ ), and information pertaining to participant eligibility will be communicated by the research assistant to the PI. On randomisation, the PI will communicate group allocation to the physiotherapist

\section{Blinding}

Outcome measures will be obtained by research assistants who will be blinded to group allocation. Interventions will be administered by a physiotherapist. The risk of unblinding is minimised by the concealed group allocation process (ie, group allocation is performed by the PI, who is not involved in the assessments or delivering the intervention). Participants are also reminded not to talk about their exercises with the research assistant in the assessment session after exercise. An additional measure of caution is that after the training is finished, we will ask the research assistant to guess group allocation for each participant, rate their confidence in their guess of group allocation and note if they had received any information to violate blinding. In cases where blinding is violated, the balance measures will be recoded from video footage by another blinded research assistant.

\section{METHODS: DATA MANAGEMENT AND ANALYSIS \\ Data collection methods}

Outcome measures and cohort descriptors will be obtained in two separate sessions by research assistants who are blinded to group allocation. Cohort descriptors, secondary outcome measures and lower extremity strength will be obtained in the first test session. $\mathrm{VO}_{\text {2peak }}$ will be obtained in the second test session (figure 1). This will be done to ensure that measures of aerobic capacity and strength are not influenced by fatigue. Primary and secondary outcome measurement will be repeated in two separate sessions at the end of the training programmes.

As this is a longitudinal study, participants may be lost to follow-up. In order to keep track of participants, we will request contact information of a friend or family member.
This information will only be used to obtain information about the whereabouts of a research participant if we are unable to contact them after multiple attempts.

\section{Data management}

Electronic data will be transferred from University of Toronto to Toronto Rehabilitation Institute for analysis and storage using secure data transfer methods. Electronic data will be stored on secure hospital servers. Electronic files containing patient names and contact information will be password protected. Hard copies of files containing deidentified data will be stored in locked cabinets and/or in offices that are locked when not occupied. Consent forms will be stored in locked cabinets/ offices separately from other data. Only those individuals who require access to the data for the purpose of this study will be provided with the password to the file containing identifiers and/or the keys to the locked cabinet/office.

\section{Statistical analysis}

Cohort descriptors and baseline values for primary and secondary outcomes will be compared between groups using Mann-Whitney $\mathrm{U}$ tests (continuous variables) or Fisher's exact test (categorical variables). Measures that differ between groups at baseline may be used as covariates in the analysis of primary and secondary outcomes. To test the primary hypothesis, we will calculate the $95 \%$ CI for the postintervention difference between groups (RBT minus AST) for the primary outcomes $\left(\mathrm{VO}_{2 \text { peak }}\right.$ and knee flexion and extension strength for each limb). Our hypothesis of non-inferiority will be supported if the lower limits of the $95 \%$ CIs are greater than the negative of the non-inferiority limits. ${ }^{71}$ We will use both intentionto-treat and per-protocol analyses to test the primary hypothesis, as is recommended ${ }^{71}$; per-protocol analysis will include only those participants who complete at least $80 \%$ of training sessions. We will also use paired t-tests for each group individually to determine if the groups improve over time in the primary outcomes. To address the secondary objectives, we use analysis of covariance (ANCOVA) to compare post-training BBS, mini-BEST and $\mathrm{ABC}$ scores between groups, with the baseline value for each measure as a covariate. The dependent variable will be rank transformed prior to conducting the ANCOVA to allow for non-parametric analysis.

\section{Sex and gender considerations}

Within the general population, women have lower cardiorespiratory fitness and muscle strength than men, ${ }^{72} 73$ but women and men can both benefit equally from aerobic and resistance training to increase physical fitness. ${ }^{72} 7475$ Likewise, women with stroke also have lower aerobic capacity ${ }^{27}$ and strength ${ }^{76-78}$ than men. There is no evidence of sex differences in adaptation to aerobic exercise poststroke. ${ }^{79}$ We are unaware of any study that examined sex differences in adaptation to resistance training poststroke. Therefore, we will conduct subgroup analysis 
by sex on strength measures to determine if such differences exist.

Research in cardiac rehabilitation suggests that women are less likely to enrol in exercise, have lower attendance and are more likely to withdraw from the programme than men ${ }^{80-82}$ These findings may be explained by gender roles (eg, low social support) rather than biological differences. ${ }^{80} 82$ Similar trends are seen in stroke rehabilitation, with slightly fewer women participating in aerobic exercise than men. ${ }^{83}$ Women may be less likely to enrol in this study, have lower attendance or be more likely to withdraw than men. We will track and report attendance and withdrawal rates by sex/gender.

\section{METHODS: MONITORING \\ Data monitoring}

A Safety and Monitoring Committee will be set up to ensure that patient safety is maintained by monitoring the trial for possible harmful effects. The members of this committee will be clinical and patient safety experts who are not involved in the study. The committee will evaluate the data for any adverse events and recommend whether the study should continue, be modified or stopped for safety concerns. The Safety and Monitoring Committee will be an independent multidisciplinary group of four to five members with experience in rehabilitation and conduct of clinical exercise trials. Membership will last until the trial is complete. The members will be free of major conflicts of interest that could prevent them from objectively reviewing the interim data and giving advice. The Safety and Monitoring Committee will meet once prior to the initiation of the trial to discuss and agree on the mandate, 6 months after initiation of the trial and then two to three times/year until the end of the study to evaluate the accrued data on adverse events. Additional meetings may be held if deemed necessary by committee members.

A Trial Steering Committee will determine the criteria for the progression of the internal pilot to the main study. ${ }^{84}$ The committee will meet one time at the start of the study and one time after the data for the internal pilot is collected and analysed. In the first meeting, the committee will develop the criteria concerning recruitment and results which will ensure the success of the main trial. In the second meeting the committee will discuss the results and will decide on the progression of the internal pilot with or without modifications.

\section{Potential harms}

\section{CPET and aerobic exercise}

There is a very low risk of injury or cardiovascular complications with exercise or testing. Pavy $e t a l^{85}$ cited event rates of 1 per 8484 exercise stress tests in cardiac rehabilitation. A recent study found no serious cardiovascular complications during 93 cardiopulmonary exercise stress tests conducted in people with stroke. ${ }^{27}$ The CPET will be overseen by a physician and we will minimise the risk of cardiac complications by monitoring ECG during the exercise test. The CPET will be stopped if one of the following occurs ${ }^{86}$ :

1. When any of following thresholds are met:

- Heart rate reaches/exceeds the age-predicted maximal heart rate.

- Cadence cannot be maintained; for example, decrease of $>10 \mathrm{rpm}$ after one reminder.

2. Additional indications to stop the test:

- Drop in systolic blood pressure by $10 \mathrm{~mm} \mathrm{Hg}$ or a failure of blood pressure to rise with increase in exercise intensity.

- Excessive rise in blood pressure.

- Failure of heart rate to increase or drop in heart rate with increased exercise intensity.

- Patient requests to stop and/or physical manifestations of severe fatigue.

- Signs of poor perfusion, for example, lightheadedness, confusion, ataxia, pallor, cyanosis, cold clammy skin, and so on.

- Onset of angina-like symptoms.

- Abnormal/severe shortness of breath.

- Failure of test equipment.

There is a risk that the tests will reveal possible health problems of which participants were previously unaware (eg, ECG abnormalities during the CPET) as previously reported. ${ }^{27}$ In this event, participants will be informed of the situation by the testing physician and will be urged to make an appointment with their doctor. With the patient's consent, the results of the CPET will be sent to their primary care physician, cardiologist and/or neurologist (if applicable).

Heart rate and blood pressure will be measured at rest at the start of every study visit to obtain a baseline measure of cardiovascular function. If measured blood pressure or heart rate is outside of an acceptable range (systolic: 90-140 mm Hg; diastolic: 60-90 mm Hg; heart rate: $60-100 \mathrm{bpm}$ ) a second measure will be obtained. If the second measurement reveals elevated heart rate and/or blood pressure, the participant will be allowed to rest seated for $5 \mathrm{~min}$, after which heart rate and blood pressure will be remeasured. If the second measurement reveals low heart rate and/or blood pressure, the participant will be offered a glass of water and heart rate and blood pressure will be remeasured after 5 min. Participants with heart rate/blood pressure measurements outside the acceptable range will also be questioned regarding recent medications (what they have taken and when, or if they have not taken their usual medications), when they last had something to eat and drink and if they recently took caffeine or exercised. The decision to continue or terminate the study visit will be made by study personnel who are members of regulated healthcare profession (ie, physiotherapist, registered kinesiologist or medical doctor) considering factors such as the participants' usual resting heart rate/blood pressure, how far the measured values are outside of the acceptable range, the participants' usual medications 
(eg, beta blockers) and the participants' perception of how they are feeling. If the visit is terminated, the study personnel may advise that the participant follow-up with his/her primary care physician. If the visit continues, the physiotherapist may choose to monitor the heart rate and blood pressure regularly throughout the visit and observe the cardiovascular response to exercise (if the visit is a training session).

\section{Balance testing and training}

As the assessment and intervention include tasks that are challenging to balance control, there is a small risk that participants will lose their balance and fall. Appropriate precautions will be taken to ensure patient safety during these tasks. Interventions will be administered by a trained and licensed physiotherapist. Assessments will be completed by trained research assistants with a health sciences background. A safety harness attached to a secure point overhead will be worn for all postural perturbations (during assessment and for participants assigned to the RBT group) to prevent a fall to the floor if the individual fails to regain stability. Additionally, the research assistant or physiotherapist can provide assistance to prevent a fall. However, even if the participant is caught by the safety harness or researcher, there is a very small chance that participants will suffer a physical injury (eg, sprain or bruise). Participants assigned to the AST group are also at risk of falling during exercise. To minimise the risk of falling, exercise will be supervised by a physiotherapist who will adopt appropriate modifications for individuals at risk of falling (eg, using seated recumbent stepping instead of treadmill walking, or performing resistance exercises in a seated position).

\section{Strength testing and training}

Participants will be advised that muscle fatigue and soreness are common with exercise, particularly new exercises, but that soreness typically resolves within 2-3 days. Fatigue will also recover in a few hours. Participants will be reminded to report any episodes of soreness to study staff.

\section{Actions taken in the event of adverse events}

In case of an adverse event requiring immediate medical attention, the study personnel will follow hospital code procedures. Depending on the nature of the adverse event, this may include calling a 'code blue', calling 911 and initiating cardiopulmonary resuscitation. As the greatest risk of cardiopulmonary resuscitation is during the CPET, consent for resuscitation will be obtained immediately before the CPET, per routine clinical procedures.

In the event of a minor physical injury, the physiotherapist will provide first aid, will advise the participant regarding follow-up with a medical professional (eg, family doctor) and home treatment (eg, rest, ice, compression, elevation) and will follow-up with the participant after a day or two.

\section{Auditing}

The PI will periodically review the completeness and accuracy of study documents to ensure the integrity of the trial. Any adverse event during assessment and exercise sessions will be recorded by the research assistants and the physiotherapist, respectively. These records will be reviewed periodically by the Safety and Monitoring Committee.

\section{ETHICS AND DISSEMINATION \\ Research ethics approval}

Research ethics approval has been received by the Research Ethics Boards of University Health Network (study ID: 18-5784.0, approved 21 June 2019) and the University of Toronto (protocol number: 37859 , approved 27 June 2019).

\section{Protocol amendments}

Substantive changes to the design or conduct of the study will require a formal amendment to the study protocol. Such substantive amendments will be agreed on by the members of the Trial Steering Committee and will be approved by the Research Ethics Boards prior to implementation. Minor administrative changes to study documents (eg, correcting a typographical error or clarifying a questionnaire item) may also be implemented, with the Research Ethics Boards notified of the changes.

\section{Consent}

The research assistant will initially discuss the study with potential participants via telephone; the research assistant will explain the study procedures and risks. If the volunteer provides verbal consent to participate in the study, the research assistant will complete an eligibility checklist. If the volunteer meets the criteria for the study, he/she will be scheduled to come to the site for an initial assessment. During the initial assessment, the research assistant will explain the study again and will obtain written informed consent to participate (online supplementary appendix). An informed consent process checklist will be completed during the initial telephone conversation and during the initial assessment.

A referral form will be used to share participant contact and health information, and any questions participants have about the CPET, with the CPET staff. Participants will be contacted via telephone by the study physician prior to the CPET to have a more detailed discussion about the risks of the CPET. The physician will answer any questions that participants have about the CPET during this telephone conversation. Participants' preference for resuscitation will also be discussed during the telephone conversation. Written consent for the CPET will be obtained by the testing physician on the participant's CPET visit. The participant will have another opportunity to have their questions answered in person at the start of this visit. Participants will be reminded that they can 
withdraw from the study if they are not comfortable with the risks of the CPET.

\section{Confidentiality}

Identifiable information (participant names and contact information) will be stored separately from health information and study data in a password-protected file, with the password only known to those individuals who are not blinded to group allocations. A participant ID number will be used to link identifiable information with health information and study data. The link between the participant ID number and name will be destroyed after data have been collected and verified. Deidentified data will be kept in a secure location for 10 years.

\section{Access to data}

All study investigators will have access to the full data set.

\section{Ancillary and post-trial care}

The institutions will be responsible for providing outof-pocket expenses to ensure that a participant receives immediate medical care in the event that the participant experiences an adverse health event (eg, injury) as a result of participation in the study.

\section{Dissemination policy}

Study participants will receive a letter of appreciation at the end of the study, which will include a summary of the study results. Study results will be submitted for publication in a peer-reviewed journal describing analysis of the primary and secondary outcomes within 6 months of completing data collection. All individuals who meet the International Committee of Medical Journal Editors criteria for authorship will be included as authors on any publications arising from this work. There is no current plan to make the participant-level data set available publicly; however, the data set may be made available in future via a Data Access Committee, if such a committee is established by the institution.

To facilitate knowledge translation after this study, we have adopted an integrated knowledge translation approach, including knowledge users (ELI, SM and PO) early in the planning stages for the study. These knowledge users have contributed to study objectives and design, and will be involved at all stages of the project. End-of-project knowledge translation to external stakeholders (eg, research community and clinicians) will occur through publication in peer-reviewed journals and presentations at conferences and workshops. We are currently in the process of developing a clinical toolkit to enable physiotherapists to complete RBT with their clients. We will update this clinical toolkit with knowledge gained from the current project.

\section{Twitter Azadeh Barzideh @Azadeh_Barzideh and Avril Mansfield @avrilmansfield}

Contributors $A B$ drafted the manuscript. $A M$ conceived the study and is the grant holder. AB, ELI, SusM, CD and AM developed the interventions. SusM, SunM, PO and AM will facilitate data collection. AB, SusM, CD, DJ, AHH, ELI, SunM, GM, PO and AM contributed to refining the study protocol and approved the final manuscript.
Funding This study is supported by the Heart and Stroke Foundation of Canada (G-18-0021807). AM holds a New Investigator Award from the Canadian Institutes of Health Research (MSH-141983).

Disclaimer The funding sources had no role in the design of this study and will not have any role during its execution, analyses, interpretation of the data or decision to submit results

Competing interests None declared.

Patient and public involvement Patients and/or the public were not involved in the design, or conduct, or reporting, or dissemination plans of this research.

Patient consent for publication Not required.

Provenance and peer review Not commissioned; externally peer reviewed.

Open access This is an open access article distributed in accordance with the Creative Commons Attribution Non Commercial (CC BY-NC 4.0) license, which permits others to distribute, remix, adapt, build upon this work non-commercially, and license their derivative works on different terms, provided the original work is properly cited, appropriate credit is given, any changes made indicated, and the use is non-commercial. See: http://creativecommons.org/licenses/by-nc/4.0/.

ORCID iD

Avril Mansfield http://orcid.org/0000-0002-0396-5815

\section{REFERENCES}

1 Gordon NF, Gulanick M, Costa F, et al. Physical activity and exercise recommendations for stroke survivors. Stroke 2004;35:1230-40.

2 Billinger SA, Arena R, Bernhardt J, et al. Physical activity and exercise recommendations for stroke survivors: a statement for healthcare professionals from the American heart Association/ American stroke association. Stroke 2014;45:2532-53.

3 Garber CE, Blissmer B, Deschenes MR, et al. American College of sports medicine position stand. quantity and quality of exercise for developing and maintaining cardiorespiratory, musculoskeletal, and neuromotor fitness in apparently healthy adults: guidance for prescribing exercise. Med Sci Sports Exerc 2011;43:1334-59.

4 Rimmer JH, Wang E, Smith D. Barriers associated with exercise and community access for individuals with stroke. J Rehabil Res Dev 2008;45:315-22.

5 Zalewski KR, Dvorak L. Barriers to physical activity between adults with stroke and their care partners. Top Stroke Rehabil 2011;18 Suppl 1:666-75.

6 Idowu OA, Adeniyi AF, Ogwumike OO, et al. Perceived barriers to physical activity among Nigerian stroke survivors. Pan Afr Med J 2015;21:274.

7 Mansfield A, Knorr S, Poon V, et al. Promoting optimal physical exercise for life: an exercise and self-management program to encourage participation in physical activity after discharge from stroke Rehabilitation-A feasibility study. Stroke Res Treat 2016;2016:9476541

8 Bonner NS, O'Halloran PD, Bernhardt J, et al. Developing the stroke exercise preference inventory (SEPI). PLoS One 2016;11:e0164120.

9 Damush TM, Plue L, Bakas T, et al. Barriers and facilitators to exercise among stroke survivors. Rehabil Nurs 2007;32:253-62.

10 Jurkiewicz MT, Marzolini S, Oh P. Adherence to a home-based exercise program for individuals after stroke. Top Stroke Rehabil 2011;18:277-84.

11 Mansfield A, Wong JS, Bryce J, et al. Does perturbation-based balance training prevent falls? systematic review and meta-analysis of preliminary randomized controlled trials. Phys Ther 2015;95:700-9.

12 Mansfield A, Peters AL, Liu BA, et al. Effect of a perturbation-based balance training program on compensatory stepping and grasping reactions in older adults: a randomized controlled trial. Phys Ther 2010;90:476-91.

13 Pai Y-C, Yang F, Bhatt T, et al. Learning from laboratory-induced falling: long-term motor retention among older adults. Age 2014;36:1367-76.

14 Bhatt T, Yang F, Pai Y-C. Learning to resist gait-slip falls: long-term retention in community-dwelling older adults. Arch Phys Med Rehabil 2012;93:557-64.

15 Bhatt T, Yang F, Pai Y-C. Learning from falling: retention of fallresisting behavior derived from one episode of laboratory-induced slip training. J Am Geriatr Soc 2011;59:2392-3.

16 Bhatt T, Pai YC. Generalization of gait adaptation for fall prevention: from moveable platform to slippery floor. $J$ Neurophysiol 2009;101:948-57. 
17 Mansfield A, Aqui A, Danells CJ, et al. Does perturbation-based balance training prevent falls among individuals with chronic stroke? a randomised controlled trial. BMJ Open 2018;8:e021510.

18 Mansfield A, Inness EL, Komar J, et al. Training rapid stepping responses in an individual with stroke. Phys Ther 2011;91:958-69.

19 Pescatello LS. ACSM's guidelines for exercise testing and prescription. Baltimore, MD: Wolters Kluwer, Lippincott Williams \& Wilkins, 2014.

20 Godin G, Shephard RJ. Godin leisure-time exercise questionnaire. Med Sci Sports Exerc 1997;29:S36-8.

21 Mansfield A, Aqui A, Centen A, et al. Perturbation training to promote safe independent mobility post-stroke: study protocol for a randomized controlled trial. BMC Neurol 2015;15:87.

22 Mansfield A, Schinkel-Ivy A, Danells CJ, et al. Does perturbation training prevent falls after discharge from stroke rehabilitation? A prospective cohort study with historical control. J Stroke Cerebrovasc Dis 2017;26:2174-80.

23 Mansfield A, Peters AL, Liu BA, et al. A perturbation-based balance training program for older adults: study protocol for a randomised controlled trial. BMC Geriatr 2007;7:12.

24 Marzolini S, Brooks D, Oh P, et al. Aerobic with resistance training or aerobic training alone poststroke: a secondary analysis from a randomized clinical trial. Neurorehabil Neural Repair 2018;32:209-22.

25 Mezzani A, Hamm LF, Jones AM, et al. Aerobic exercise intensity assessment and prescription in cardiac rehabilitation: a joint position statement of the European association for cardiovascular prevention and rehabilitation, the American association of cardiovascular and pulmonary rehabilitation and the Canadian association of cardiac rehabilitation. Eur J Prev Cardiol 2013;20:442-67.

26 Borg GA. Psychophysical bases of perceived exertion. Med Sci Sports Exerc 1982;14:377-81.

27 Marzolini S, Oh P, Mcllroy W, et al. The feasibility of cardiopulmonary exercise testing for prescribing exercise to people after stroke. Stroke 2012;43:1075-81.

28 Faber MJ, Foster C, Greany J, et al. Comparison of Bruce and modified Bruce treadmill protocols. J Cardiopulm Rehabil 2005;25:290.

29 Garner KK, Pomeroy W, Arnold JJ. Exercise stress testing: indications and common questions. Am Fam Physician 2017;96:293-9.

30 Eng JJ, Dawson AS, Chu KS. Submaximal exercise in persons with stroke: test-retest reliability and concurrent validity with maximal oxygen consumption. Arch Phys Med Rehabil 2004;85:113-8.

31 American Thoracic Society, American College of Chest Physicians. ATS/ACCP statement on cardiopulmonary exercise testing. Am J Respir Crit Care Med 2003;167:211-77.

32 Marzolini S, Oh P, Mcllroy W, et al. The effects of an aerobic and resistance exercise training program on cognition following stroke. Neurorehabil Neural Repair 2013;27:392-402.

33 Boyne P, Reisman D, Brian M, et al. Ventilatory threshold may be a more specific measure of aerobic capacity than peak oxygen consumption rate in persons with stroke. Top Stroke Rehabil 2017:24:149-57.

34 Kuo H-K, Leveille SG, Yen C-J, et al. Exploring how peak leg power and usual gait speed are linked to late-life disability: data from the National health and nutrition examination survey (NHANES), 19992002. Am J Phys Med Rehabil 2006;85:650-8.

35 Flansbjer U-B, Miller M, Downham D, et al. Progressive resistance training after stroke: effects on muscle strength, muscle tone, gait performance and perceived participation. $J$ Rehabil Med 2008:40:42-8.

36 Flansbjer U-B, Holmbäck AM, Downham D, et al. What change in isokinetic knee muscle strength can be detected in men and women with hemiparesis after stroke? Clin Rehabil 2005;19:514-22.

37 Rabelo M, Nunes GS, da Costa Amante NM, et al. Reliability of muscle strength assessment in chronic post-stroke hemiparesis: a systematic review and meta-analysis. Top Stroke Rehabil 2016;23:26-35.

38 Tripp EJ, Harris SR. Test-Retest reliability of isokinetic knee extension and flexion torque measurements in persons with spastic hemiparesis. Phys Ther 1991;71:390-6.

39 Pohl PS, Startzell JK, Duncan PW, et al. Reliability of lower extremity isokinetic strength testing in adults with stroke. Clin Rehabil 2000;14:601-7.

40 Eng JJ, Kim CM, Macintyre DL. Reliability of lower extremity strength measures in persons with chronic stroke. Arch Phys Med Rehabil 2002;83:322-8.

41 Clark DJ, Condliffe EG, Patten C. Reliability of concentric and eccentric torque during isokinetic knee extension in post-stroke hemiparesis. Clin Biomech 2006;21:395-404.
42 Noorizadeh Dehkordi S, Talebian S, Olyaei G, et al. Reliability of isokinetic normalized peak torque assessments for knee muscles in post-stroke hemiparesis. Gait Posture 2008;27:715-8.

43 Hody S, Croisier J-L, Bury T, et al. Eccentric muscle contractions: risks and benefits. Front Physiol 2019;10:536.

44 Berg Ket al. Measuring balance in the elderly: preliminary development of an instrument. Physiotherapy Canada 1989;41:304-11.

45 Franchignoni F, Horak F, Godi M, et al. Using psychometric techniques to improve the balance evaluation systems test: the miniBESTest. J Rehabil Med 2010;42:323-31.

46 Blum L, Korner-Bitensky N. Usefulness of the Berg balance scale in stroke rehabilitation: a systematic review. Phys Ther 2008;88:559-66.

47 Tsang CSL, Liao L-R, Chung RCK, et al. Psychometric properties of the Mini-Balance evaluation systems test (Mini-BESTest) in community-dwelling individuals with chronic stroke. Phys Ther 2013;93:1102-15.

48 Powell LE, Myers AM. The Activities-specific balance confidence (ABC) scale. J Gerontol A Biol Sci Med Sci 1995;50A:M28-34.

49 Botner EM, Miller WC, Eng JJ. Measurement properties of the Activities-specific balance confidence scale among individuals with stroke. Disabil Rehabil 2005;27:156-63.

50 Eng JJ, Chu KS, Dawson AS, et al. Functional walk tests in individuals with stroke: relation to perceived exertion and myocardial exertion. Stroke 2002;33:756-61.

51 Salbach N, Bayley M, Brooks D, et al. A guide to an evidenceinformed approach to using the 10-metre and 6-minute walk tests post-stroke. secondary a guide to an evidence-informed approach to using the 10-metre and 6-minute walk tests post-stroke, 2018. Available: https://www.physicaltherapy.utoronto.ca/wp-content/ uploads/2018/09/iWalk-Guide-Canadian-Version-Posted17Sep2018.pdf

52 Flansbjer U-B, Holmbäck AM, Downham D, et al. Reliability of gait performance tests in men and women with hemiparesis after stroke. $J$ Rehabil Med 2005;37:75-82.

53 Jones CJ, Rikli RE, Beam WC. A 30-S chair-stand test as a measure of lower body strength in community-residing older adults. Res $Q$ Exerc Sport 1999;70:113-9.

54 Rikli RE, Jones CJ. Functional fitness normative scores for Community-Residing older adults, ages 60-94. J Aging Phys Act 1999;7:162-81.

55 Lyders Johansen K, Derby Stistrup R, Skibdal Schjøtt C, et al. Absolute and Relative Reliability of the Timed 'Up \& Go' Test and '30second Chair-Stand' Test in Hospitalised Patients with Stroke. PLoS One 2016;11:e0165663.

56 Hyndman D, Ashburn A, Stack E. Fall events among people with stroke living in the community: circumstances of falls and characteristics of fallers. Arch Phys Med Rehabil 2002;83:165-70.

57 Lamb SE, Jørstad-Stein EC, Hauer K, et al. Development of a common outcome data set for fall injury prevention trials: the prevention of falls network Europe consensus. J Am Geriatr Soc 2005;53:1618-22.

58 Washburn RA, Zhu W, McAuley E, et al. The physical activity scale for individuals with physical disabilities: development and evaluation. Arch Phys Med Rehabil 2002;83:193-200.

59 Trigg R, Wood VA. The subjective index of physical and social outcome (SIPSO): a new measure for use with stroke patients. Clin Rehabil 2000;14:288-99.

60 Yates JS, Lai SM, Duncan PW, et al. Falls in community-dwelling stroke survivors: an accumulated impairments model. J Rehabil Res Dev 2002;39:385-94.

61 Ebrahim S, Thompson PW, Baskaran V, et al. Randomized placebocontrolled trial of brisk walking in the prevention of postmenopausal osteoporosis. Age Ageing 1997;26:253-60.

62 Obembe AO, Eng JJ. Rehabilitation interventions for improving social participation after stroke: a systematic review and meta-analysis. Neurorehabil Neural Repair 2016;30:384-92.

63 Goldstein LB, Bertels C, Davis JN. Interrater reliability of the NIH stroke scale. Arch Neurol 1989;46:660-2.

64 Gowland C, Stratford P, Ward M, et al. Measuring physical impairment and disability with the Chedoke-McMaster stroke assessment. Stroke 1993;24:58-63.

65 Kasner SE. Clinical interpretation and use of stroke scales. Lancet Neurol 2006;5:603-12.

66 Flight L, Julious SA. Practical guide to sample size calculations: noninferiority and equivalence trials. Pharm Stat 2016;15:80-9.

67 Sealed Envelope Ltd. Power calculator for continuous outcome noninferiority trial., 2012. Available: https://www.sealedenvelope.com/ power/continuous-noninferior/ [Accessed 25 Jul 2017]. 
68 Piaggio G, Elbourne DR, Pocock SJ, et al. Reporting of noninferiority and equivalance randomized trials: extension of the CONSORT 2010 statement. J Am Geriatr Soc 2012;308:2594-604.

69 Jin $\mathrm{H}$, Jiang $\mathrm{Y}$, Wei Q, et al. Effects of aerobic cycling training on cardiovascular fitness and heart rate recovery in patients with chronic stroke. NeuroRehabilitation 2013;32:327-35.

70 Tang A, Marzolini S, Oh P, et al. Factors associated with change in aerobic capacity following an exercise program for individuals with stroke. J Rehabil Med 2013;45:32-7.

71 Schumi J, Wittes JT. Through the looking glass: understanding noninferiority. Trials 2011;12:106.

72 McArdle WD, Katch FI, Katch VL, et al. Nutrition, and Human Performance. 5th edn. Philadelphia: Lippincott Williams \& Wilkins, 2001.

73 Yanovich R, Evans R, Israeli E, et al. Differences in physical fitness of male and female recruits in gender-integrated army basic training. Med Sci Sports Exerc 2008;40:S654-9.

74 Symons TB, Vandervoort AA, Rice CL, et al. Effects of maximal isometric and isokinetic resistance training on strength and functional mobility in older adults. J Gerontol A Biol Sci Med Sci 2005;60:777-81.

75 Paterson DH, Jones GR, Rice CL. Ageing and physical activity: evidence to develop exercise recommendations for older adults. Can J Public Health 2007;98 Suppl 2:S69-108.

76 Ryan AS, Ivey FM, Serra MC, et al. Sarcopenia and physical function in middle-aged and older stroke survivors. Arch Phys Med Rehabil 2017;98:495-9.

77 Flansbjer U-B, Downham D, Lexell J. Knee muscle strength, gait performance, and perceived participation after stroke. Arch Phys Med Rehabil 2006;87:974-80.

78 Carvalho C, Sunnerhagen KS, Willén C. Walking performance and muscle strength in the later stage poststroke: a nonlinear relationship. Arch Phys Med Rehabil 2013:94:845-50.

79 Baert I, Daly D, Dejaeger E, et al. Evolution of cardiorespiratory fitness after stroke: a 1-year follow-up study. Influence of prestroke patients' characteristics and stroke-related factors. Arch Phys Med Rehabil 2012;93:669-76.

80 Daly J, Sindone AP, Thompson DR, et al. Barriers to participation in and adherence to cardiac rehabilitation programs: a critical literature review. Prog Cardiovasc Nurs 2002;17:8-17.

81 Samayoa L, Grace SL, Gravely S, et al. Sex differences in cardiac rehabilitation enrollment: a meta-analysis. Can J Cardiol 2014;30:793-800.

82 Grace SL, Abbey SE, Shnek ZM, et al. Cardiac rehabilitation II: referral and participation. Gen Hosp Psychiatry 2002;24:127-34.

83 Prout EC, Brooks D, Mansfield A, et al. Patient characteristics that influence enrollment and attendance in aerobic exercise early after stroke. Arch Phys Med Rehabil 2015;96:823-30.

84 Avery KNL, Williamson PR, Gamble C, et al. Informing efficient randomised controlled trials: exploration of challenges in developing progression criteria for internal pilot studies. BMJ Open 2017;7:e013537.

85 Pavy B, lliou MC, Meurin P, et al. Safety of exercise training for cardiac patients: results of the French registry of complications during cardiac rehabilitation. Arch Intern Med 2006;166:2329-34.

86 PROPEL research group. Submaximal graded exercise assessment guidelines secondary submaximal graded exercise assessment guidelines, 2018. Available: https://events.myconferencesuite.com/ images/UHN/docs/593/Submaximal_Graded_Exercise_Assessment Guidelines_vJun.pdf [Accessed 16 Jun 2018]. 